\title{
Length changes and texture prediction during free end torsion test of copper bars with FEM and remeshing techniques
}

\author{
Laurent Duchêne $^{\mathrm{a}}$, Fouad El Houdaigui ${ }^{\mathrm{b}, 1}$, Anne Marie Habraken ${ }^{\mathrm{b}}$ \\ ${ }^{\mathrm{a}}$ Department $C O B O$, Royal Military Academy, Avenue de la Renaissance 30, 1000 Brussels, Belgium ${ }^{\mathrm{b}}$ Department ArGEnCo, Division MS ${ }^{2}$ F, \\ University of Liège, Chemin des Chevreuils 1, 4000 Liège, Belgium
}

\begin{abstract}
FEM numerical simulations of the experimental free end torsion tests [Toth, L.S., Jonas, J.J., Daniel, D., Bailey, J.A., 1992. Texture development and length changes in copper bars subjected to free end torsion. Textures Microstruct. 19, 245-262] of copper cylindrical bars were analysed in the present study. The self-made Finite Element (FE) code LAGAMINE was used to compute numerical prediction of the Swift effect, i.e. the lengthening of the cylinder during the torsion. The material behaviour was represented by an interpolation law [Habraken, A.M., Duchêne, L., 2004. Anisotropic elasto-plastic finite element analysis using a stress-strain interpolation method based on a polycrystalline model. Int. J. Plasticity 20 (8-9), 1525-1560] linked with a Taylor poly-crystal plasticity model either based on a strain rate insensitive or a visco-plastic crystal plasticity model. The influence of texture evolution was analysed. A torsion dedicated remeshing technique was developed to allow very large strain simulations. Predicted axial lengthening and predicted textures were compared to experimental measurements. A good agreement was obtained for shear strain up to 2.0. The Swift effect related angular shift of the pole figure maxima from symmetrical orientations was reproduced correctly.
\end{abstract}

Keywords: B. Anisotropic material; B. Crystal plasticity; C. Finite elements; Swift effect

\section{Introduction}

The torsion of cylindrical bars or tubes is of great importance for the characterization of material properties in the large strain regime. Indeed, under adequate experimental settings, torsion tests remain stable up to very large strains before failure. Contrarily, tensile tests generally become unstable for large strains due to necking, while compression tests are influenced by friction and require large mechanical efforts.

However, it is well known that the material behaviour during torsion is different from the behaviour during tensile or compression tests. Bearing in mind that plastic strains involve dislocation glides which are linked to shearing at the crystal level, the different material behaviours can be explained by the cone of maximum shear planes present in compression and tension compared to the only two maximum shear planes in torsion (Miller and McDowell, 1996b).

Hopefully, crystal plasticity models, by nature, can account for this different material behaviour in torsion compared to tension or compression.

A particular phenomenon observed during torsion under certain conditions is the Swift effect. It refers to the lengthening or shortening of a cylinder or a tube submitted to large strain torsion in the plastic strain regime. The Swift effect has first been observed by Swift (1947).

Lengthening during torsion was previously studied by Poynting $(1909,1912)$ in the elastic regime. This torsion induced lengthening was found by Weber et al. (2000) to have a purely geometrical origin.

Contrarily, a key point concerning the Swift effect is the crystallographic texture evolution due to the large plastic strains involved during these torsion tests. Texture measurements have been carried on before and during experimental torsion processes (see e.g. Toth et al., 1992, 1991; Qods et al., 2005). Beside, plastic anisotropy

\footnotetext{
${ }^{1}$ On the point of leaving.
} 
models based on texture evolution have been applied for the Swift effect prediction of twisted materials (Toth et al., 1990; Wu, 2003; Wu et al., 1996; Qods et al., 2005; Toth and Jonas, 1989). From these studies, it is admitted that the initial texture of the material and especially the texture developed during the torsion test have a crucial influence on the Swift effect.

As shown by Toth et al. (1988) and Weber et al. (2000), the rigid body rotation associated to the shearing induced by the torsion process yields to an asymmetric crystal orientation distribution. In turn, the axes of the yield locus are slightly tilted away from the symmetry axes of the tube or the cylinder (Rohatgi et al., 1995; Jonas et al., 1998). The resulting material behaviour is lengthening during torsion processes.

On the other hand, the shortening observed under particular experimental conditions can be attributed to dynamic recrystallization during torsion (Toth et al., 1992; Rohatgi et al., 1995; Weber et al., 2000).

In order to observe lengthening or shortening of the cylinder or the tube, displacements along its axial direction must be permitted during the torsion process without any axial forces in the specimen. Specific experimental devices are therefore required. This loading is called free end torsion (Khan et al., 2006).

Analogous axial effect exists in the case of fixed end torsion. As length variations of the tube are not permitted, axial stress develops. Free end torsion and fixed end torsion are related phenomena but they must be analysed separately as they involve important differences in the boundary conditions of testing.

The goal of this study was to model accurately the free end torsion of copper bars at room temperature. The prediction of the Swift effect and the texture evolution are compared to experimental results from Toth et al. (1992).

Even if the torsion process studied presents axial and circumferential symmetries, a nonlinear three-dimensional FE analysis with an actual mesh of the whole bar was investigated. This method accounts for the radial distribution of the shear strain linked to the torsion in the bar. The interaction between the different radial layers of the cylinder and the resulting lengthening were computed. For this purpose, the Swift effect prediction requires very accurate material's behaviour modelling in the large plastic strain regime; not only for the constitutive model but also for the whole FEM approach (element, remeshing technique).

A recently developed finite element was utilized. Its formulation is based on the new shear locking treatment proposed by Wang and Wagoner (2004) and it makes use of an original method for the determination of the material axes.

Since it appeared to be a crucial point for the Swift effect prediction, a crystal plasticity constitutive model adapted for the texture evolution computation was employed for this study (Habraken and Duchêne, 2004).

Finally, a very important feature of the present work was the use of a remeshing technique especially dedicated to the free end torsion of cylindrical bars. Remeshing was imposed by the very large strains involved in the studied process.

\section{Swift effect reported in the literature}

Fig. 1 presents the first experimental results concerning torsion induced elongation strains in the plastic regime. They have been reported by Swift (1947) for seven metals.

Author's observations were: "It will be seen that all seven metals show a general tendency to elongate under severe torsional strain. (...) The final elongation varies from about 1 per cent, for a carbon steel to over 11 per cent."

Furthermore, Swift noticed that lengthening was larger for fcc than for bcc metals.

According to Swift (1947), the Swift effect has its origin in the interaction between crystals with different orientations. The shearing induced by the torsion is assumed to be accommodated at the crystal level by dislocation glide along well defined slip systems. The hardening behaviour on these slip systems depends on the orientation of every crystal with respect to the applied shear strain. High hardened and low hardened grains (depending on their initial orientation) can progressively be distinguished during the torsion. The interaction between these hard and soft grains induces their reorientation, i.e. texture evolution. According to Swift's 
assumptions, hard grains tend to align their length with the axial direction of the tube or cylinder while soft grains preferably align perpendicular to the axial direction. These reorientations are subsequently assumed to be at the origin of the observed torsion induced lengthening.

This explanation of the Swift effect was, as stated by the author, largely speculative; it was indeed later found to be invalid. Unexpectedly, it is anyway in agreement with some experimental observations. First, it can explain why fcc metals are more subjected to lengthening than bcc metals. The description of Swift (1947) being closely linked to slip system activity, a lower number of permitted slip systems in each crystal emphasises the phenomenon. Secondly, at high temperatures, a shortening of the tube or cylinder is observed experimentally. The explanation of Swift is based on the work hardening in each crystal; at high temperatures, dynamic recrystallization occurs and annihilates the work hardening based extension. Furthermore, a non-hardening material like lead is not subjected to the Swift effect even at room temperature. Whereas, room temperature is too low to permit dynamic recrystallization in steel, aluminium or copper for instance and thus only lengthening is observed.

Fig. 1: Experimental extension strain as a function of shear strain for 70-30 brass (a), stainless steel (b), aluminium (c), cupro-nickel (d), copper (e), mild-steel ( $f$ ), 0.5\% carbon steel ( $g$ ), from Swift (1947).

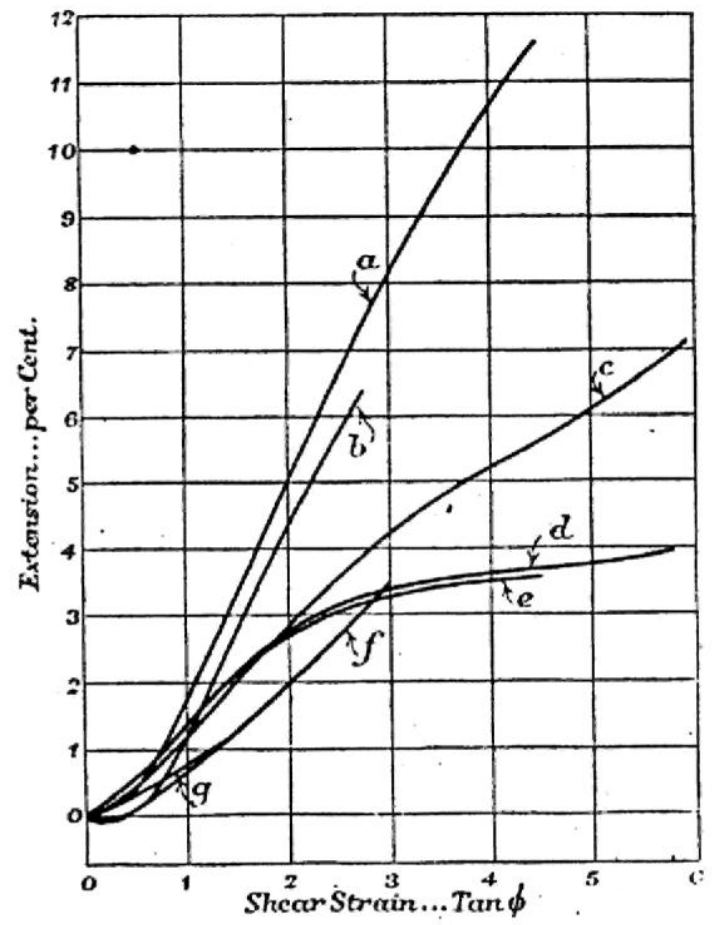

The effect of sample temperature during free end torsion of copper bars was analysed experimentally in Toth et al. (1992). Lengthening was observed during the torsion at room temperature and $125^{\circ} \mathrm{C}$. Lengthening followed by shortening appeared for higher temperatures $\left(200^{\circ} \mathrm{C}\right.$ and $\left.300{ }^{\circ} \mathrm{C}\right)$, see Fig. 2 . By analysing the texture of the samples at different shear strains, it appeared that the classical texture components observed during shearing were present for the cases at room temperature and $125^{\circ} \mathrm{C}$. These texture components were responsible for the lengthening. For the two higher temperatures, evidences of dynamic recrystallization appeared after a critical strain. This dynamic recrystallization was at the origin of the shortening behaviour observed in Toth et al. (1992) during the torsion tests at high temperatures.

The effect of the initial texture on the Swift effect was analysed by Toth et al. (1991) during torsion of copper wires. Different initial textures were obtained by annealing at different temperatures. The particular texture components responsible for the lengthening and shortening were identified. 
Miller and McDowell (1996a) and Miller and McDowell (1996b) reported a 7\% elongation strain during torsion of 304L stainless steel. This elongation strain was obtained for a torsion induced shear strain of 2.5.

Fig. 2: Lengthening during free end torsion of copper bars at different temperatures $\left({ }^{\circ} \mathrm{C}\right)$, from Toth et al. (1992).

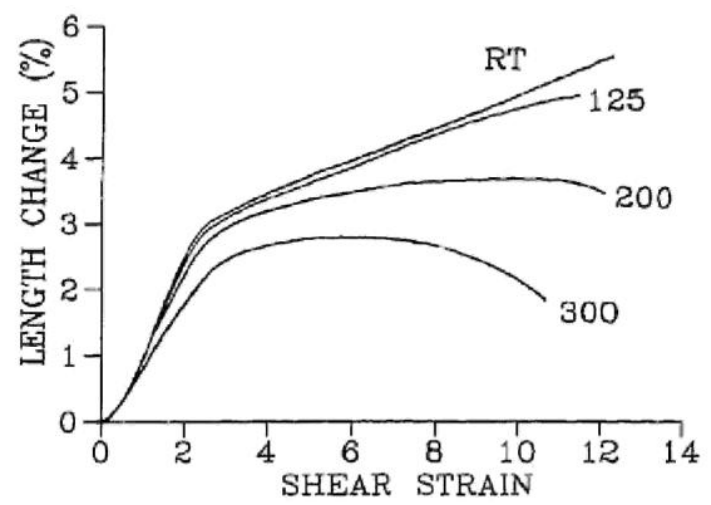

Several authors have developed and applied numerical models for the Swift effect prediction during torsion simulations.

- In Toth et al. (1990), the unconstrained shear of thin walled tubes was simulated using Sachs' hypothesis. Effect of texture evolution was taken into account. The effect of initial texture on the lengthening was analysed in details in Toth and Jonas (1989) by simulating the experiences of Rose and Stüwe (1968), where especially designed thin walled tubes with different strong textures were used.

According to Toth et al. (1990) and Toth and Jonas (1989), the strain rate sensitivity has a very large influence on the Swift effect. Toth et al. (1990) observed lengthening for low values of strain rate sensitivity $(m<0.13)$, shortening for high values $(0.167<m<1)$ and lengthening followed by shortening for intermediate values of $m$. - Xiao et al. (2001) developed two numerical models dedicated to the torsion of thin walled cylindrical tubes. The first model is an elastic perfectly plastic model with the logarithmic rate while the second is a kinematic hardening plastic model with logarithmic rate. The results of the second model with two resolution algorithms are compared to experimental results in Fig. 3.

- Wu (2003) developed a model based on the quadratic Hill (1948) yield locus using a contravariant stress formulation with an observer independent yield function. The rotation of material texture was computed. They predicted an elongation strain of $1.3 \%$ during the free end torsion of an aluminium tube.

Fig. 3: Swift effect: axial elongation versus the shear strain (extracted from Xiao et al., 2001).

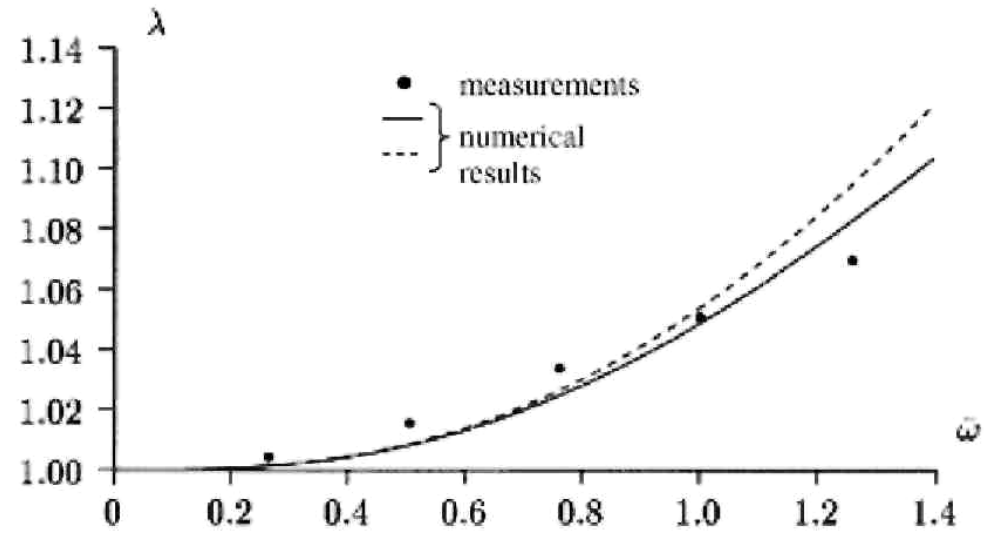


From a numerical point of view, it is more complicated to model a cylinder than a tube. Indeed, if the wall thickness of the tube is small enough compared to its mean radius, the analysis of a tube can be reduced to the analysis of its mid-thickness layer. On the other hand, the radial dependency of the material state must be fully taken into consideration for the analysis of a cylinder. Nevertheless, some research works proposed numerical solutions for the Swift effect prediction during torsion of cylinders.

- Wu et al. (1996) developed a torsion dedicated FE model where each finite element has the shape of a tube. The total FE mesh, consisting of tubes fitting into each other, yielded to the cylinder. This FE technique was coupled with a visco-plastic model based on Taylor polycrystalline plasticity. The influence of the hardening model on the numerical results was particularly focussed on. At the level of the crystal, a slip system activity based evolution law for the strain hardening state was employed. Latent and self-hardening were distinguished and four single slip hardening laws were compared.

- An alternative technique was recently proposed by Qods et al. (2005). The cylindrical bar is also divided into tubes but no FE formulation is used. They compared a method based on the equilibrium equation with a method based on the minimum plastic power for the prediction of the axial strain during torsion. A polycrystal viscoplastic Taylor code computed the texture development and the stress state in each tube. A large effect of the strain rate sensitivity was noticed.

Different formulations are reported in the literature concerning the torsion induced shear strain. For instance, four definitions of large shear strain are presented in Toth et al. (1990). The following one was used in the present paper:

$$
\gamma=\frac{r \Phi}{l_{0}}=r \Psi
$$

where $r$ is the current radius, $\Phi$ is the twist angle, $l_{0}$ is the initial length and $\Psi$ is the twist angle per unit initial length of the cylinder or the tube.

An equivalent strain formulation valid for small and large strains was furthermore proposed by Shrivastava et al. (1982):

$$
\varepsilon_{\mathrm{eq}}=\frac{\gamma}{\sqrt{3}}
$$

When the torsion of a tube is analysed, some authors (see e.g. Fig. 3 from Xiao et al., 2001) use the shear strain $\bar{\omega}$ defined as:

$$
\bar{\omega}=\frac{1}{2} \psi \bar{R} \approx \frac{1}{2} \gamma
$$

where $\bar{R}$ is the initial mean radius of the tube.

Table 1 summarizes some experimental results concerning free end torsion tests. Several materials and geometries have been tested. Depending on experimental conditions, a wide range of elongation strains were reported from shortening to lengthening. Some Swift effect predictions obtained with different models are presented in Table 2. Analytical models refer to an analytical computation of the kinematics of the free end torsion, i.e. without FE analysis.

\section{Experimental free end torsion}

For this study, the experimental free end torsion of pure copper cylinders presented in Toth et al. (1992) was analysed. The samples were 14 or $8 \mathrm{~mm}$ long and their radius was $3.2 \mathrm{~mm}$.

A mean grain size of the copper around $30 \mu \mathrm{m}$ was obtained by Toth et al. (1992) as a result of a heat treatment of $550{ }^{\circ} \mathrm{C}$ in vacuum during $30 \mathrm{~min}$.

The torsion was achieved up to a shear strain y of 12 (at the surface of the cylinders) at different temperatures ranging from room temperature to $300{ }^{\circ} \mathrm{C}$. During the tests, the applied torque, the twist angle and the length change were measured continuously. From these measurements, the lengthening as a function of the shear strain 
is plotted in Fig. 2. It was observed that the torsion at room temperature and $125^{\circ} \mathrm{C}$ induced lengthening of the bars while lengthening followed by shortening appeared for the tests at $200{ }^{\circ} \mathrm{C}$ and $300{ }^{\circ} \mathrm{C}$.

Texture measurements were achieved after the torsion of the bars at different applied shear strains. X-ray technique was used. The initial texture of the samples was also measured experimentally. The $\{111\}$ and $\{100\}$ pole figures of the initial texture are shown in Fig. 4 in the radial $(r)$, circumferential $(\vartheta)$ and axial $(z)$ reference system. The measured initial texture was the average texture on the volume of the sample and, due to the bar forming processes, it presented a cylindrical symmetry. Besides, a weak anisotropy was noticed.

The textures of the samples submitted to torsion at high temperature $\left(300{ }^{\circ} \mathrm{C}\right)$ proved the activation of dynamic recrystallization. This phenomenon is at the origin of the shortening observed at large shear strains.

Table 1: Experimental Swift effect reported in the literature

\begin{tabular}{|c|c|c|c|c|c|}
\hline $\begin{array}{l}\text { Shear } \\
\text { strain }\end{array}$ & $\begin{array}{l}\text { Elongation strains } \\
(\%)\end{array}$ & Materials investigated & $\begin{array}{l}\text { Crystal } \\
\text { structure }\end{array}$ & $\begin{array}{l}\text { Samples } \\
\text { geometry }\end{array}$ & References \\
\hline Up to 6 & From 1 to 11.5 & $\begin{array}{l}70-30 \text { brass, stainless steel, } \\
\text { aluminium, cupro-nickel, copper, } \\
\text { mild steel and } 0.5 \% \text { carbon steel }\end{array}$ & $\begin{array}{l}\text { fec and } \\
\text { bcc }\end{array}$ & Tubes & Swift (1947) \\
\hline $0.2-0.3$ & $0.4-0.8$ & Iron and steel & bcc & Wires & $\begin{array}{l}\text { Rohatgi et al. } \\
\text { (1995) }\end{array}$ \\
\hline Up to 5 & $\begin{array}{l}\text { From }-4 \text { to } 3 \\
\text { depending on the } \\
\text { annealing } \\
\text { temperature }\end{array}$ & Copper & fcc & Wires & $\begin{array}{l}\text { Toth et al. } \\
\text { (1991) }\end{array}$ \\
\hline 12 & up to 5.5 & Copper at different temperatures & fcc & Bars & $\begin{array}{l}\text { Toth et al. } \\
\text { (1992) }\end{array}$ \\
\hline 0.15 & $\begin{array}{l}\text { from }-1.2 \text { to }+1.3 \\
\text { depending on the } \\
\text { initial texture }\end{array}$ & Copper & fcc & $\begin{array}{l}\text { Thin-walled } \\
\text { tubes }\end{array}$ & $\begin{array}{l}\text { Rose and Stüwe } \\
\text { (1968) }\end{array}$ \\
\hline 2.6 & 7 & 304L stainless steel & fcc & $\begin{array}{l}\text { Thin-walled } \\
\text { tubes }\end{array}$ & $\begin{array}{l}\text { Miller and } \\
\text { McDowell } \\
(1996 a, b)\end{array}$ \\
\hline 0.5 & 1.6 & Aluminium & fcc & $\begin{array}{l}\text { Thin-walled } \\
\text { tubes }\end{array}$ & Wu (2003) \\
\hline
\end{tabular}

Fig. 4: $\{111\}$ and $\{100\}$ pole figures of copper bar initial texture, from Toth et al. (1992).
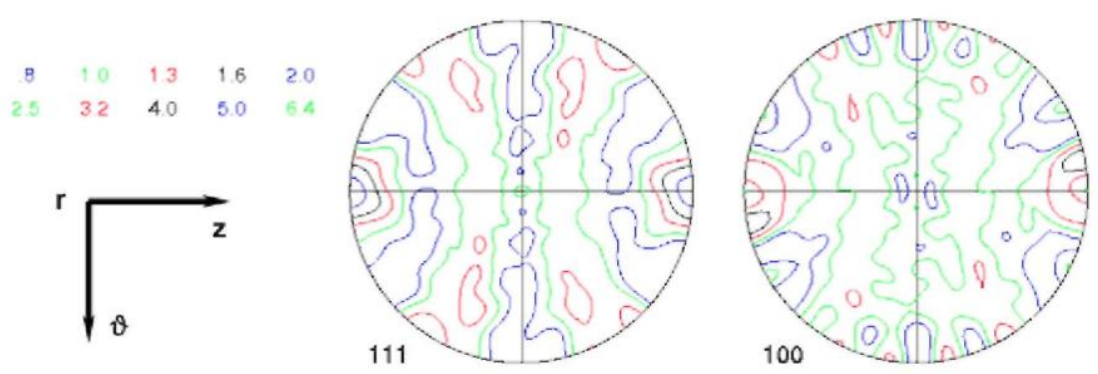
Table 2: Simulated elongation strains reported in the literature

\begin{tabular}{|c|c|c|c|c|c|c|}
\hline $\begin{array}{l}\text { Shear } \\
\text { strain }\end{array}$ & $\begin{array}{l}\text { Elongation strains } \\
(\%)\end{array}$ & Methods used & $\begin{array}{l}\text { Materials } \\
\text { investigated }\end{array}$ & $\begin{array}{l}\text { Crystal } \\
\text { structure }\end{array}$ & Geometry & References \\
\hline Up to 5 & $\begin{array}{l}\text { From }-10 \text { to } 16 \\
\text { depending on the } \\
\text { initial texture }\end{array}$ & $\begin{array}{l}\text { Analytical rate sensitive } \\
\text { polycrystal Sachs model }\end{array}$ & Copper & fcc & Wires & $\begin{array}{l}\text { Toth et al. } \\
(1991)\end{array}$ \\
\hline 5 & $\begin{array}{l}-3 \text { to } 9 \text { depending } \\
\text { on the initial texture } \\
\text { and the strain rate } \\
\text { sensitivity }\end{array}$ & $\begin{array}{l}\text { Analytical polycrystal } \\
\text { viscoplastic model using } \\
\text { equilibrium equation }\end{array}$ & Copper & fcc & Wires & $\begin{array}{l}\text { Toth et al. } \\
(2005)\end{array}$ \\
\hline 1.5 & $\approx 10$ & $\begin{array}{l}\text { Torsion dedicated FE } \\
\text { analysis with a viscoplastic } \\
\text { Taylor-type polycrystal } \\
\text { model and sophisticated } \\
\text { hardening laws }\end{array}$ & Copper & fcc & Bars & $\begin{array}{l}\text { Wu et al. } \\
(1996)\end{array}$ \\
\hline 8 & $\begin{array}{l}\text { Up to } 10 \text { depending } \\
\text { on the strain rate } \\
\text { sensitivity }\end{array}$ & $\begin{array}{l}\text { Two analytical models } \\
\text { with a polycrystal } \\
\text { viscoplastic Taylor code }\end{array}$ & Copper & fcc & Bars & $\begin{array}{l}\text { Qods et al. } \\
(2005)\end{array}$ \\
\hline 0.15 & $\begin{array}{l}\text { From }-1.5 \text { to }+1.5 \\
\text { depending on the } \\
\text { initial texture }\end{array}$ & $\begin{array}{l}\text { Two analytical models: } \\
\text { rate sensitive and } \\
\text { continuum mechanics of } \\
\text { textured polycrystals }\end{array}$ & Copper & fcc & $\begin{array}{l}\text { Thin- } \\
\text { walled } \\
\text { tubes }\end{array}$ & $\begin{array}{l}\text { Toth and } \\
\text { Jonas (1989) }\end{array}$ \\
\hline Up to 20 & $\begin{array}{l}\text { From }-35 \text { to }+50 \\
\text { depending on the } \\
\text { strain rate } \\
\text { sensitivity }\end{array}$ & $\begin{array}{l}\text { Analytical rate sensitive } \\
\text { crystal plasticity model of } \\
\text { the Sachs' type }\end{array}$ & $\begin{array}{l}\text { Initially } \\
\text { isotropic } \\
\text { material }\end{array}$ & fcc & $\begin{array}{l}\text { Thin- } \\
\text { walled } \\
\text { tubes }\end{array}$ & $\begin{array}{l}\text { Toth et al. } \\
(1990)\end{array}$ \\
\hline 0.5 & 1.3 & $\begin{array}{l}\text { Analytical model based on } \\
\text { Hill (1948) }\end{array}$ & Aluminium & fcc & $\begin{array}{l}\text { Thin- } \\
\text { walled } \\
\text { tubes }\end{array}$ & Wu (2003) \\
\hline 2.8 & 711 & $\begin{array}{l}\text { Analytical elastic-perfectly } \\
\text { plastic model Analytical } \\
\text { kinematic hardening } \\
\text { plastic model }\end{array}$ & Metal & & $\begin{array}{l}\text { Thin- } \\
\text { walled } \\
\text { tubes }\end{array}$ & $\begin{array}{l}\text { Xiao et al. } \\
(2001)\end{array}$ \\
\hline
\end{tabular}

Fig. 5: Shear stress-shear strain curves during free end torsion of copper bars at different temperatures $\left({ }^{\circ} \mathrm{C}\right)$, from Toth et al. (1992).

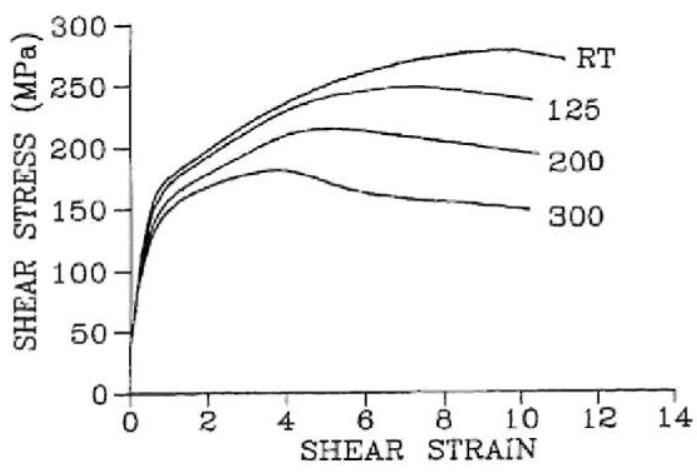

During this study, in order to avoid dynamic recrystallization observed at high temperature (and not modelled in our simulations), the experimental results at room temperature were focussed on.

Fig. 5 presents the shear stress-shear strain curves deduced from the torque measurements using Nadai (1950) formula. These curves were used to determine the hardening behaviour of the studied material. 
The power-type hardening law of Eq. (4) was fitted on the experimental curve at room temperature. In Eq. (4), the equivalent stress $\sigma$ is assumed to be a function of the equivalent plastic strain $\varepsilon^{p} . C$, $\varepsilon_{0}$ and $n$ are material parameters. The values corresponding to the studied copper bars at room temperature are presented in Eq. (5)

$$
\begin{aligned}
& \sigma=C\left(\varepsilon_{0}+\varepsilon^{p}\right)^{n} \\
& \left\{\begin{array}{l}
C=334.19 \mathrm{MPa} \\
\varepsilon_{0}=0.001561 \\
n=0.2208
\end{array}\right.
\end{aligned}
$$

\section{Finite element modelling}

The goal of this study is to use FE technique with LAGAMINE for the numerical modelling of free end torsion. The observations of Toth et al. (1992) in terms of length changes and texture evolution are taken as reference. The numerical tools investigated for this purpose are described hereafter.

\subsection{FE code LAGAMINE}

The numerical simulations described in the present paper were achieved thanks to the self-made FE code LAGAMINE. It is an implicit non-linear FE code with a Lagrangian formulation and it is adapted to large strains and large displacements. The kinematic of the code, developed in order to ensure objectivity, is described hereafter. The development of this code began in 1984 for rolling simulations (Habraken et al., 1998). Since then, it has been applied to numerous other forming processes, such as forging (Habraken and Cesc-otto, 1990), continuous casting (Castagne et al., 2004), deep drawing (Duchêne et al., 2002; Duchêne and Habraken, 2005), powder compaction (Mosbah et al., 1999) and cooling processes (Habraken and Bourdouxhe, 1992; Casotto et al., 2005). LAGAMINE has a large element library (see for instance Cescotto and Charlier, 1993; Zhu and Cescotto, 1994; Habraken and Cescotto, 1998), as well as numerous constitutive laws (Zhu and Cescotto, 1995; Habraken and Duchêne, 2004). The particular finite element and the constitutive laws used in the present study are further described in next sections.

\subsection{BWD3D finite element}

The BWD3D element is an 8-node 3D brick element with a mixed formulation adapted to large strains and large displacements. It uses a reduced integration scheme (with only one integration point) and an hourglass control technique. This element is based on the non-linear three-field (stress, strain and displacement) HU-WASHIZU variational principle with the "assumed strain method" (Simo and Hughes, 1986; Belytschko and Bind-eman, 1991). The BWD3D has recently been implemented in the FE code LAGAMINE.

A first feature of the BWD3D element is a new shear locking treatment based on the Wang and Wagoner (2004) method. This method identifies the hourglass modes responsible for the shear locking and removes them. The two bending hourglass modes and the warp (non-physical) hourglass mode are eliminated. The volumetric locking treatment is also based on the elimination of inconvenient hourglass modes.

A second feature of this new element is the use of a corotational reference system. In order to be able to identify the hourglass modes (which is a crucial point of the method), the formulation of the element kinematic must be expressed in a corotational reference system (Belytschko and Bindeman, 1991), closely linked to element coordinates. This reference system must have its origin at the center of the element and its reference axes are aligned (as much as possible, depending on the element shape) with element edges. A grateful consequence of this corotational reference system is a simple and accurate treatment of the hourglass stress objectivity, by using initial and final time step rotation matrices.

The shear locking and the volumetric locking methods proposed by Wang and Wagoner, associated with the corotational reference system, have been successfully implemented in the BWD3D element of LAGAMINE FE code. The Wang-Wagoner method, contrarily to some other shear locking methods (see e.g. Li and Cescotto, 1997), has deep physical roots which makes it very efficient for various FE analyses. Further details about the hourglass and the locking treatments in the BWD3D element can be found in Duchêne et al. (2005a). Up to now, the BWD3D element has proved its superiority compared to the former version of this element: the BLZ3D having another shear locking treatment (Zhu and Cescotto, 1994) during deep drawing simulations, incremental forming and the large strain torsion processes presented in this study (see Section 5 and Duchêne et al., 2005b). 
This proves the large influence of the accuracy of the element formulation and in particular its shear locking treatment on FE results (see also Alves de Sousa et al., 2007).

Another important aspect of the BWD3D element is the method used to determine the local reference axes. These local axes are a convenient way to fulfil the objectivity of the stress integration and, in addition, to model correctly the anisotropic material behaviour during the large rotations involved in FE simulations (Choi et al., 2006). Note that this local reference system used for objectivity and material modelling is completely independent from the corotational reference system used to identify the hourglass modes.

The way to choose the local reference system is based on the Constant symmetric local velocity gradient method developed by Cescotto and Munhoven (see Cescotto, 1984; Munhoven and Habraken, 1995). This method concerns the computation of the velocity gradient, whose symmetric part is the well-known strain rate tensor and the skew-symmetric part is the spin tensor, related to rigid body rotation.

The implementation of a non-linear constitutive law in a large strain FE code implies a step by step procedure. The integration of the kinematic equations must be achieved incrementally. Consequently, during the computation of one FE time step, the velocity field at the beginning of the step is known and an estimation of the velocity field at the end of the step is assumed. However, the strain path between these two configurations is not determined. Different assumptions concerning the strain path have been examined by several authors, see e.g. Hughes (1983) and Ponthot (2002).

According to the Constant symmetric local velocity gradient method, the velocity gradient is assumed to be constant during the time step and this assumption imposes the strain path. Also the constant value of the velocity gradient can be determined from the configurations at the beginning of the step and at the end of the step.

If it is expressed in the global reference system (which remains always fixed during the FE simulation), the so obtained constant velocity gradient is generally non-symmetric. The symmetry of the velocity gradient can, however, be obtained by expressing it in another reference system. In other words, the symmetry condition of this method fixes the choice of the local reference system. As a consequence, the spin tensor corresponding to the symmetric constant velocity gradient is equal to zero if it is expressed in the local reference system. By the way, the integration of the constitutive law in the local axes is incrementally objective without the necessity of Jaumann type corrections. Furthermore, the local reference system is also assumed to correspond to the material axes into which material properties are defined.

\subsection{MINTY3 constitutive law}

It is well known that plastic deformation of polycrystalline material induces reorientation of individual grains into preferred orientations. This phenomenon, i.e. the texture evolution, is responsible for induced mechanical anisotropy of the material (Beyerlein and Tomé, 2006), which plays an important role in forming processes. The implementation of texture evolution into FE codes is therefore of great importance. Unfortunately, micro-macro models generally require very long computation time and large memory storage.

These considerations led to the development of the local yield locus approach MINTY3 (Microscopic INTerpolated Yield locus 3D) able to predict texture evolution during FE modelling of industrial forming processes. With this model, only a small zone of the yield locus is computed. This zone is updated when its position is no longer located in the part of interest in the yield locus or when the yield locus changes due to texture evolution. So this approach strongly limits the number of calls to the micro-macro module which activates a high number of single crystal computations. It can be considered the same as the use of a macroscopic law with some updating of anisotropic parameters based on microscopic models. As shown in Duchêne (2003), it reduced the computation time by a factor of around 60 compared to a systematic call of the Taylor polycrystal model during a deep drawing simulation.

This local yield locus approach has already been validated and extensively used to model deep drawing processes (see Duchêne, 2003; Habraken and Duchêne, 2004 for details).

This model is specific in the sense that it does not use a yield locus formulation either for plastic criterion nor in the stress integration scheme. A linear stress-strain interpolation in the five-dimensional (5D) stress space described by the following equation is used at the macroscopic scale:

$$
\underline{\sigma}=\tau \underline{\underline{C}} \cdot \underline{u}
$$


In this equation, $\sigma$ is a $5 \mathrm{D}$ vector containing the deviatoric part of the stress; the hydrostatic part being elastically computed according to a linear elasticity law. The $5 \mathrm{D}$ vector $\underline{u}$ is the deviatoric plastic strain rate direction (it is a unit vector), $\tau$ is the so-called critical resolved shear stress. It is a scalar which describes the work hardening according to the exponential relationship of Eq. (7) where the strength coefficient $K$, the offset $\Gamma^{0}$ and the hardening exponent $n$ are material parameters fitted on experimental data and $\Gamma$ is the polycrystal induced slip.

$$
\tau=K\left(\Gamma^{0}+\Gamma\right)^{n}
$$

The macroscopic anisotropic interpolation is included in matrix $\underline{\underline{C}}$ of Eq. (6). Its identification relies on five directions: $\underline{u}_{i}(i=1, \ldots, 5)$ advisedly chosen in the deviatoric strain rate space and their associated deviatoric stresses: $\underline{\sigma}_{i}(i=1, \ldots, 5)$ computed by the polycrystal plasticity model. The micro-macro model considered here makes use of Taylor's assumption of equal macroscopic plastic strain and microscopic crystal plastic strain. It computes the average of the response of a set of representative crystals evaluated with a microscopic model taking into account the plasticity at the level of the slip systems. In this paper, two versions of the microscopic Full Constraints (FC) Taylor's model are investigated: one coupled with a rate insensitive single crystal plasticity model (Habraken and Duchêne, 2004) and one coupled with a visco-plastic single crystal model (see Toth et al., 1988; Qods et al., 2005; Toth et al., 2005). A significant influence of the strain rate sensitivity on the Swift effect prediction was noticed (see Qods et al., 2005 and Sections 2 and 5 of this paper).

The stress vectors $\underline{\sigma}_{i}$ lie on the yield locus according to the crystal plasticity model. They define the vertices of the interpolation domain and are called 'stress nodes'. The $\underline{\underline{C}}$ matrix is built on the basis of these 5 stress nodes.

With this method, only a small part of the yield locus is known. As long as the interpolation is achieved in the domain delimited by these 5 stress nodes, the interpolation matrix $\underline{\underline{C}}$ is valid. When the stress direction explored during FE computation falls out of this domain, updating of the stress nodes must take place; a new interpolation matrix is then computed.

The above considerations are sufficient to understand the basic concepts of the local yield locus implemented in the LAGAMINE code. Further details and properties of such parameterisation of an $N$-dimensional space have been investigated in Duchêne (2003) and Habraken and Duchêne (2004).

Material's texture is represented at each integration point of the FE mesh by a set of crystallographic orientations. These orientations are computed from initial texture measurements using the MTM-FHM software (Van Houtte, 1994). The influence of the number of crystals has largely been investigated in Duchêne et al. (2000); it turns out that a number of 2000 crystals is a good compromise between accuracy and computation time.

Texture evolution is computed by Taylor's model on the basis of the strain history for each integration point. The set of crystallographic orientations of each integration point is subsequently updated to represent the current material's texture during the FE simulation. As it induces an evolution of the material's behaviour, when texture evolution takes place, the $\underline{C}$ matrix describing the current local yield locus must be updated. Interested readers can refer to Habraken and Duchêne (2004) for further details about the strategy used for texture evolution.

In the numerical results (see Section 5), 'MINTY3' refers to the local yield locus approach without computation of texture evolution (the initial texture is then used throughout the FE simulation) while 'EVOL' refers to the same approach but with activation of the texture evolution.

Material parameters used in the present study match to the material exploited in Toth et al. (1992) and described in Section 3 . Young's modulus was $130 \mathrm{GPa}$ (copper), Poisson's ratio was 0.34 .

The macroscopic hardening behaviour described by Eq. (4) can be adapted to the microscopic law of Eq. (7) thanks to the so-called Taylor factor. This transformation is necessary for MINTY3 constitutive law. The hardening parameters presented in Eq. (5) became:

$$
\left\{\begin{array}{l}
K=82.7 \mathrm{MPa} \\
\Gamma^{0}=0.0049 \\
n=0.2208
\end{array}\right.
$$

The 12 slip systems $\{111\}<110$ 〉 typical for fee materials were used in the crystal plasticity models. 


\subsection{FE mesh}

A particular attention was paid to the FE mesh used for the torsion simulations (several different meshes were previously tested). The constitutive law used requires large computation time especially when the texture evolution is activated. The total computation time for one simulation is more or less proportional to the number of finite elements of the mesh. On the other hand, an accurate prediction of the Swift effect requires a detailed modelling and above all an adequately refined FE mesh.

The FE mesh consisted of 12 layers (along the axial direction) of 128 elements, as shown in Fig. 6. That means a total of 1536 BWD3D elements.

The torsion was imposed in the FE code by fixing the global coordinates $X, Y$ and $\mathrm{Z}$ of the lower layer nodes and by twisting progressively the upper layer. The displacements along $X$ and $Y$ of the upper layer nodes are such that the upper layer is subjected to a rigid body rotation around the $\mathrm{Z}$-axis. In order to model the free end torsion condition, the axial (along $\mathrm{Z}$ ) degree of freedom of the twisted nodes remained free.

In this torsion process, the initial geometry of the bar, its material properties and the applied load were such that circumferential symmetry (along the angular position in cylindrical coordinates) and axial symmetry of the results were expected. All the simulation results (stress, texture...) should only depend on the radial coordinate. According to that idea, simplified meshes were tested but unfortunately, correct boundary conditions could not be reached. The small shortcomings on the boundary conditions always yielded to inaccurate results.

For instance, the 12 element layers were necessary to avoid edge effect. Indeed, the upper and the lower layers were subjected to geometrical constraints necessary to impose the torsion of the cylinder; the main geometrical constraint being a fixed radius of these layers. And these geometrical constraints were likely to induce inaccurate results. The results computed at the mid-height of the cylinder were therefore the most accurate ones (compared to other layers).

Fig. 6: Initial FE mesh of the cylindrical bar.

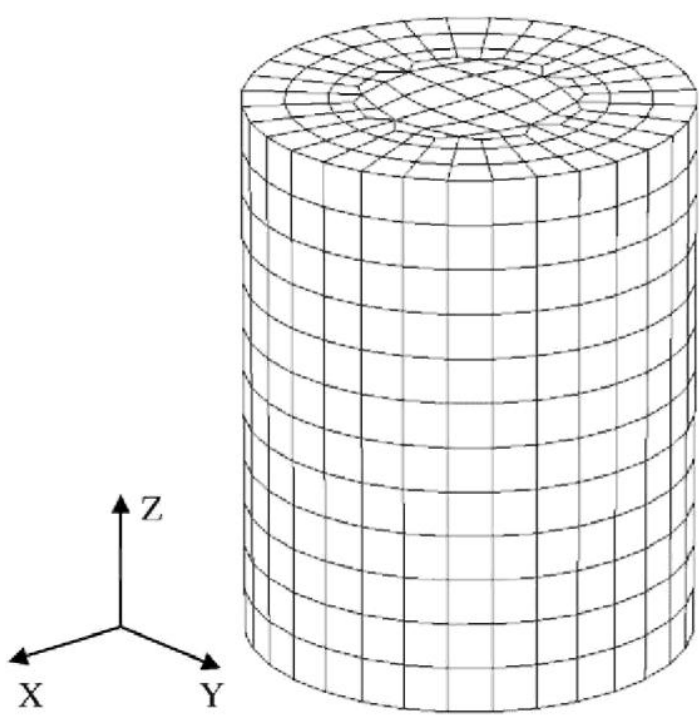

\subsection{Remeshing scheme}

In order to be able to achieve FE numerical simulation of torsion up to large strains, a remeshing technique adapted to the cylindrical torsion had to be developed. The remeshing scheme is described hereafter:

- An initial mesh of the cylinder is generated in accordance with the initial geometry of the bar described in Section 3. The distribution of the elements (number of layers and number of elements in each layer) is presented in Section 4.4.

- Initial values are defined at each integration point of the FE mesh for:

- the state variables of the material. These variables describe the current state of the material according to the 
deformation undergone. They mainly concern hardening. Generally, the initial state of the material is characterized by state variables equal to zero,

- the stress tensor; initial value is zero,

- the local axes; due to the cylindrical symmetry of the bar, the initial local axes $\underline{\mathrm{e}}_{1}, \underline{\mathrm{e}}_{2}$, and $\underline{\mathrm{e}}_{3}$ are placed along the circumferential, axial and radial directions, respectively,

- the texture; the initial texture was measured by X-ray diffraction. Again, due to the cylindrical symmetry of the bar, symmetry of its texture was observed along the axial and circumferential directions (i.e., the texture gradient along these directions is zero). Concerning the texture gradient along the radial direction, the initial measured texture corresponded to an average one. Therefore, the initial texture can be considered uniform and must be expressed in cylindrical coordinates. As, in the model, the texture is defined in the local uniform initial texture provides the desired axi-symmetric texture (in accordance with the measurements).

- A first run of the FE code is achieved up to a relatively low shear strain value on the external surface of the cylinder (compared to the shear strain expected at the end of the torsion process). The value of the shear strain at the end of this FE run must be low enough to avoid large distortions of the finite elements, which would induce inaccurate results.

- After the first FE run, a remeshing of the cylinder is achieved. A new regular perfectly cylindrical mesh is generated. In order to avoid edge effects, its geometrical parameters, i.e. its radius and its height, are based on the mid-height layer values at the end of the previous FE run. The mean radius and the mean thickness of the mid-height layer are used. The structure of the new mesh (number of layers and number of elements in each layer) is identical to the initial one. So, the number of nodes and elements for the FE simulation remains unchanged after remeshing.

- Once the new mesh is generated, the mapping of the variables of the old mesh into the new mesh must be done. As the structure of the new mesh is identical to the old one, the new mesh is located (and particularly rotated) in such a way that the elements of the mid-height layer are placed as close as possible to their old location. In this way, the elements of the mid-height layer simply correspond to themselves in the old and the new meshes.

As a consequence, two different element categories are distinguished. For the elements in the mid-height layer, the state variables describing the current material behaviour are just kept constant during the mapping. As it is expressed in the global axes (which are always unchanged), the stress tensor is not modified. The local axes of the elements, used to express the material anisotropy, are also directly copied from the old mesh to the new one. Next, the texture, which can evolve during FE simulations, is unchanged during the mapping. The texture is expressed in the local axes of each element. Finally, a particular attention must be paid to the hourglass stresses. They are essential for the description of the stress gradient inside each element. Their definition is linked to the element nodal coordinates through the intrinsic coordinates of the element (see Duchêne et al., 2005a for further details about hourglass stresses). During the remeshing, the nodal coordinates evolve. So, the mapping of the hourglass stresses is not straightforward. Anyway, due to the symmetry of the torsion process, it can be reasonably assumed that the stress gradients along the circumferential and axial axes are negligible. The corresponding hourglass stresses are subsequently supposed to be equal to zero. Only the stress gradient along the radial direction subsists. Gratefully, the intrinsic coordinate of the element along the radial direction is not altered during the remeshing (thanks to geometrical considerations). Therefore, the definition of the corresponding hourglass stress is still valid after remeshing and the mapping reduces to a simple copy from the old mesh to the new one. For the elements outside the mid-height layer, the state variables, the stress tensor, the local axes, the texture and the hourglass stresses of the associated element in the mid-height layer are exploited. • At this stage, the next FE run can be started. In fact, successive partial FE runs and remeshing computations are managed up to the desired final shear strain. The preceding procedure is then repeatedly applied.

The remeshing frequency can be characterized by the amount of shear strain imposed on the external surface of the cylinder during one FE run of the torsion simulation. A too high value yields to inaccuracy due to the distortion of the finite elements. On the other hand, low values of shear strain imply numerous remeshing computations and increase computation time. An intermediate value must be chosen. Gratefully, except for very high values of the shear strain during one FE run (larger than 0.5), the effect of the remeshing frequency on the results is almost negligible. Values ranging from 0.02 to 0.4 yielded to satisfactory results. Hereafter, a value of the shear strain during each FE run around 0.2 was generally adopted.

\section{Results and discussion}

The experimental free end torsion presented in Section 3 was modelled with the finite element method presented in Section 4. The accuracy of the finite element used as well as the constitutive laws was assessed by comparing the predicted Swift effect and the predicted textures with experiment. The influence of the texture evolution during the simulations was analysed. The results provided by the micro-macro Taylor's model either linked with 
the rate insensitive single crystal plasticity law or the visco-plastic one were compared. Finally, the effect of the remeshing technique was studied.

\subsection{Length change}

Fig. 7 presents the axial lengthening of the cylinder, i.e. the Swift effect, as a function of the torsional shear strain defined by Eq. (1). FE results obtained with the polycrystal plasticity constitutive law, without texture evolution (curve MINTY3) and with texture evolution (curves EVOL) were compared with experimental results from Toth et al. (1992).

The axial strain predicted with computation of the texture evolution by the rate insensitive Taylor's model was close to the experimental result for shear strain up to 2.0. For larger shear strain, the slope change observed on experimental curve around $\gamma=2.5$ was not obtained numerically.

Fig. 7: Axial strain versus shear strain $\gamma$ during free end torsion (experimental curve from Toth et al., 1992). $\alpha$ is the shift angle of the pole figure maxima from symmetrical orientations.

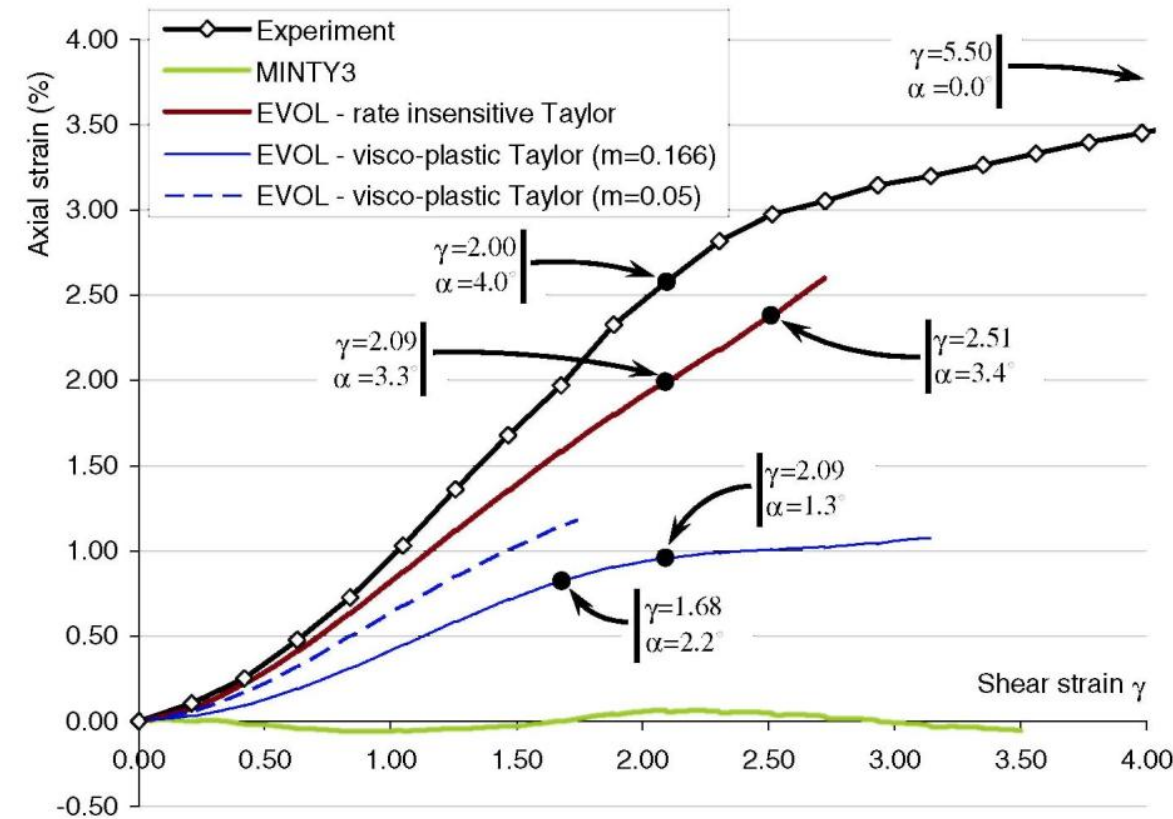

The effect of polycrystal plasticity model can be visualized by comparing the strain rate insensitive Taylor's model (Habraken and Duchêne, 2004) with the visco-plastic Taylor's model (Toth et al., 1988) which used rate sensitivity parameters $m$ equal to 0.166 and 0.05 . The viscous effect tended to reduce the amplitude of the Swift effect (particularly for the larger $m$ value). Such an effect of strain rate sensitivity has already been employed by several authors (e.g. Toth et al., 1990; Toth and Jonas, 1989; Qods et al., 2005). Beside the poor Swift effect's amplitude prediction, the visco-plastic Taylor's model was able to model the slope change experimentally observed (for $m=0.166$ ). As explained in Section 5.2, this issue is directly linked to texture evolution modelling through the orientation of the pole figure maxima (angle $\alpha$ in Fig. 7).

When the initial texture was not updated throughout the simulation (curve MINTY3), no lengthening was observed. The initial texture is indeed expected not to produce any Swift effect. For this material, it is the texture development induced by the torsion process which is responsible for the Swift effect, not the initial texture. As explained in the next section, the symmetry of the initial texture pole figures (see Fig. 4) prevents lengthening.

The influence of the remeshing procedure is presented in Fig. 8. The results obtained with the rate insensitive Taylor's model with computation of texture evolution using a remeshing frequency corresponding to an amount of shear strain of 0.2 during each FE run were compared to results using a higher remeshing frequency ( 0.1 shear strain between remeshing computations). It appeared that the remeshing frequency had a low effect on the predicted axial lengthening. 
However, for torsion simulations without remeshing, accurate results were obtained for shear strain up to 1.0. But, for larger shear strains, the lengthening was largely overestimated with or without texture evolution. This behaviour is due to an inaccuracy for very large strains induced by an excessive distortion of the finite elements.

Fig. 8 also compares the results obtained with the BWD3D element and its former version: the BLZ3D element, for the case with texture evolution and no remeshing. It appears that the axial strain predicted with the BLZ3D was completely wrong for shear strains larger than 0.5 .

Fig. 8: Axial strain versus shear strain $\gamma$ during free end torsion (experimental curve from Toth et al., 1992). Numbers in parentheses correspond to the amount of shear strain during one FE run before remeshing occurs.

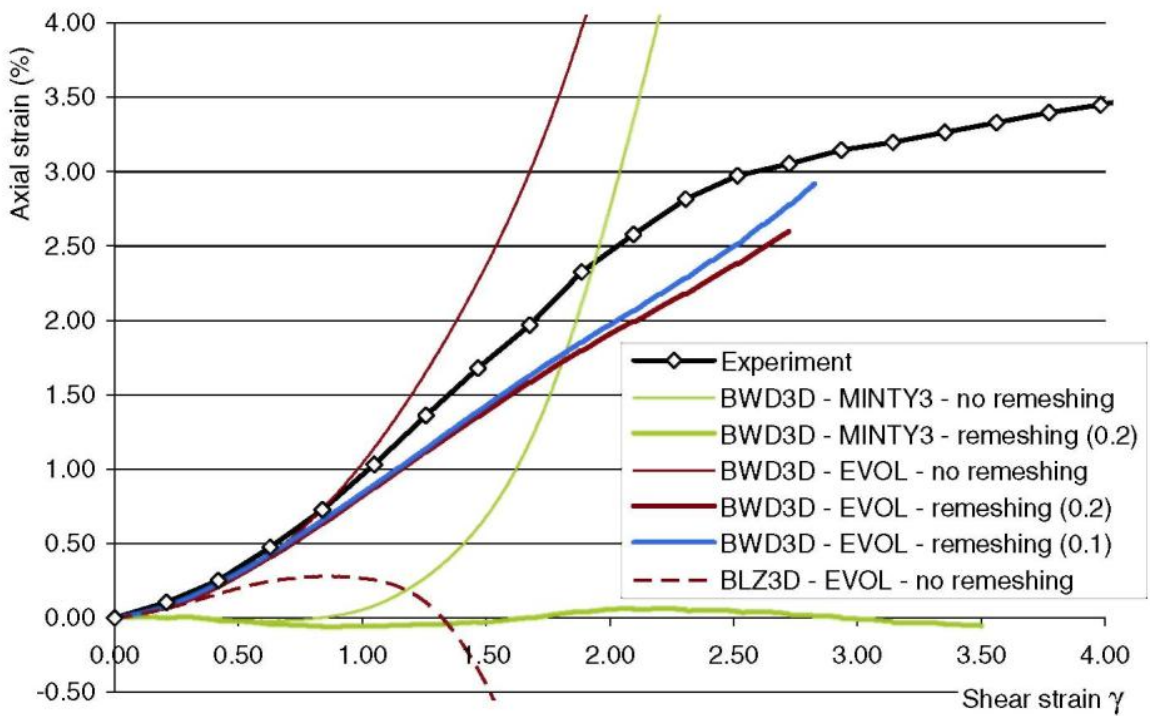

\subsection{Texture analysis}

As the texture and particularly the texture evolution are crucial points for the Swift effect, the predicted textures were analysed. During the FE simulations, the texture was updated separately for every element of the mesh, every 10 time steps. This parameter must be fixed by the FE user and a value of 10 was a good compromise between accuracy and computation time, in accordance with the time increment used during the simulations (one time increment corresponded to a shear strain increment of $0.14 \%$ ). Indeed, the material behaviour evolves smoothly during the FE simulations as a result of the texture evolution. Accordingly, the accuracy of the model would not be significantly improved with a larger updating frequency.

Fig. 9 compares the predicted texture with the measured one on a deformed sample for a shear strain of 2.0. $\{111\}$ pole figures were investigated as is usual for shear textures. The textures obtained for a shear strain of 2.0 were quite different from the initial texture (Fig. 4). For numerical simulations, the predicted textures on the external surface of the cylinder, at mid-height were considered (Fig. 9). The agreement between the predicted and the measured textures was quite good (qualitatively as well as quantitatively). It can be noticed that the predicted texture with the rate insensitive Taylor's model was slightly better than with the visco-plastic one (for $m=0.166$ ). Secondary maxima on the horizontal axis (axial coordinate of the cylinder) were indeed overestimated by the visco-plastic Taylor's model.

An angular shift $(\alpha)$ between the maxima of the pole figures and the horizontal axis corresponding to symmetry positions with respect to the sample axes can be noticed on the experimental and the predicted textures in Fig. 9. The observed shift was in the opposite direction from the shear strain. A shift of $4.0^{\circ}$ was measured on the experimental pole figure, while angles of $3.3^{\circ}$ and $1.3^{\circ}$ were observed for the rate insensitive and the viscoplastic Taylor's models, respectively. These values of the shift $\alpha$ for a shear strain of 2.0 as well as values for other shear strains are reported in Fig. 7. 
Fig. 9: Comparison of the predicted textures with experiment for a shear strain of 2.0 in $\{111\}$ pole figures.

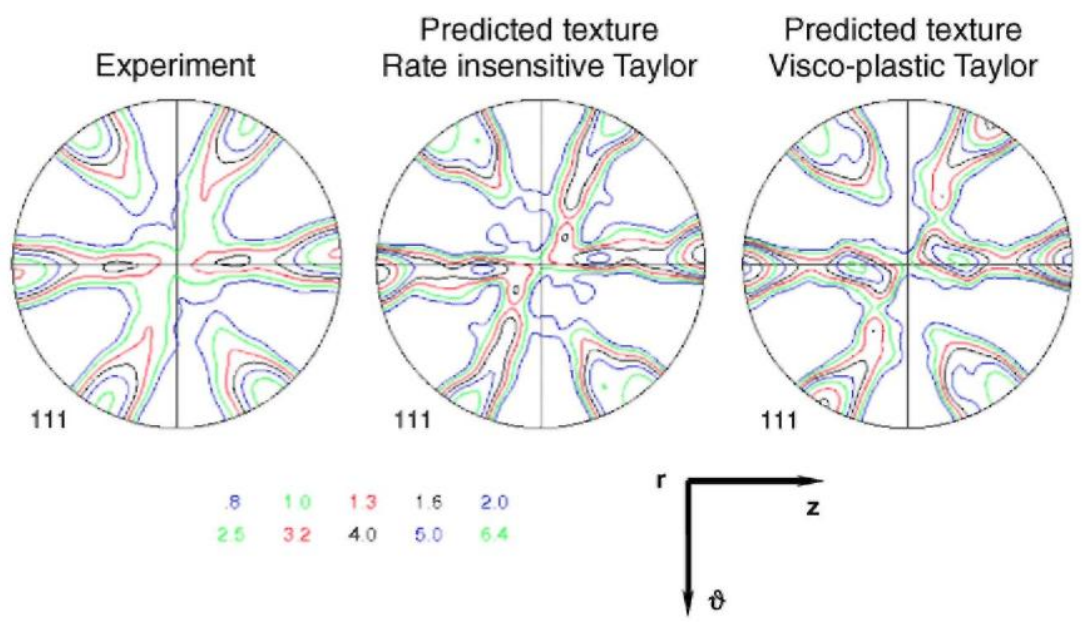

As detailed in Toth et al. (1992) and Toth et al. (1990), these small shifts are very important and closely linked to the Swift effect. They correspond to an increase of the texture components responsible for the lengthening behaviour (compared to shortening components). As a consequence, the slope of the axial lengthening curve (Fig. 7) is related to the value of this angular shift. During the experimental torsion process, the shift was $4.0^{\circ}$ for a shear strain of 2.00 and vanished for $\gamma=5.5$ (according to Qods et al., 2005); this explains the slope change observed in the experimental lengthening curve. With the rate insensitive Taylor's model, the shift remained around $3.3^{\circ}$ for shear strains up to 2.51 and therefore the slope of the curve was constant. Similar observations have been reported in Qods et al. (2005) when low strain rate sensitivity parameters have been used. Contrarily, the visco-plastic Taylor's model with $m=0.166$ predicted a shift of $2.2^{\circ}$ for a shear strain of 1.68 and the shift dropped to $1.3^{\circ}$ for $\gamma=2.09$. As a result, the slope change of the lengthening curve was modelled.

\section{Conclusions}

In this paper, the experimental results of Toth et al. (1992) for free end torsion of copper bars were predicted with polycrystal plasticity models implemented in the FE code LAGAMINE. Predicted Swift effect (axial lengthening) as a function of shear strain (see Fig. 7) was in good agreement with experimental results for shear strains up to 2.0. This is in agreement with the results presented in Qods et al. (2005) and is closely linked to the texture evolution. When a rate insensitive Taylor's model was used, the slope change observed in experimental curve was not reproduced. On the other hand, the visco-plastic Taylor's model allowed to model correctly the slope change of the axial lengthening curve but the amplitude of the predicted Swift effect was too low.

Obviously, texture evolution must be taken into account for Swift effect prediction. The angular shift of the texture maxima from the symmetrical orientations observed on the pole figures (Fig. 9) was identified to be related to the lengthening of the cylinder (see Toth et al., 1992, 1990). As this shift was reproduced correctly with the FE method, effective Swift effect prediction was managed in this study.

The plastic anisotropy induced by the texture evolution was assumed to be the unique origin of the Swift effect. As an isotropic phenomenological hardening law was employed for all the simulations, the influence of the hardening model was not analysed. Interested readers can refer to Wu et al. (1996) who focussed on the effects of the hardening laws (at the level of the slip systems) on the torsion induced lengthening.

Due to the very large strains involved during the investigated process, a remeshing technique dedicated to torsion simulations had to be developed and coupled with the FE code. Even if carrying out 3D FE computation seems complicated and computation time consuming compared with analytical models (e.g. Qods et al., 2005), a great advantage of FE models is their large adaptability. This Swift effect prediction allows validating not only the constitutive laws but also the finite elements used, their local axes choice, the remeshing technique... Complex geometries, complex texture distributions can therefore be investigated.

Even if the predicted textures obtained with the Taylor's models (rate insensitive or visco-plastic) were quite good (see Fig. 9), future developments concerning the Swift effect study will be the use of other crystal plasticity models to improve the accuracy of the predicted texture evolution. The Taylor's model is indeed known to 
generally overestimate the predicted textures. This is a consequence of the full constraints condition, which has a large influence on the slip system activity, which, in turn, influences the texture evolution. Different micromacro models have already been tested by Duchêne et al. (2004) for the torsion of a tube. The advanced Lamel (Van Houtte et al., 2005) and the self-consistent (Berbenni et al., 2007) models will be investigated.

\section{Acknowledgements}

A.M. Habraken is mandated by the National Fund for Scientific Research (Belgium). Professor Paul Van Houtte is acknowledged for providing us with texture treatment modules. The authors acknowledge Professor Laszlo Toth for the experimental data and the visco-plastic Taylor code. The authors also thank the Belgian Federal Science Policy Office (Contract P5/08) for its financial support.

\section{References}

Alves de Sousa, R.J., Yoon, J.W., Cardoso, R.P.R., Fontes Valente, R.A., Gracio, J.J., 2007. On the use of a reduced enhanced solid-shell (RESS) element for sheet forming simulations. Int. J. Plasticity 23 (3), 490-515.

Belytschko, T., Bindeman, L.P., 1991. Comput. Methods Appl. Mech. Engrg. 88, 311-340.

Berbenni, S., Favier, V., Berveiller, M., 2007. Impact of the grain size distribution on the yield stress of heterogeneous materials. Int. J. Plasticity 23 (1), 114-142.

Beyerlein, I.J., Tomé, C.N., 2006. Modeling transients in the mechanical response of copper due to strain path changes. Int. J. Plasticity. doi:10.1016/j.ijplas.2006.08.00.

Casotto, S., Pascon, F., Habraken, A.M., Bruschi, S., 2005. Thermo-mechanical-metallurgical model to predict geometrical distortions of rings during cooling phase after ring rolling operations. Int. J. Mach. Tools Manufacture 45, 657-664.

Castagne, S., Pascon, F., Bles, G., Habraken, A.M., 2004. Developments in finite element simulations of continuous casting. J. Phys. IV 120, $447-155$.

Cescotto, S., 1984. Finite deformation of solids. In: Hartley, P., Pillinger, I., Sturgess, C. (Eds.), Numerical Modelling of Material Deformation Processes. Springer-Verlag, pp. 20-67.

Cescotto, S., Charlier, R., 1993. Frictional contact finite element based on mixed variational principles. Int. J. Numer. Methods Engrg. 36, $1681-1701$.

Choi, Y., Han, C.-S., Lee, J.K., Wagoner, R.H., 2006. Modeling multi-axial deformation of planar anisotropic elasto-plastic materials, Part I: Theory. Int. J. Plasticity 22 (9), 1745-1764.

Duchêne, L., 2003. FEM study of metal sheets with a texture based, local description of the yield locus. Ph.D. Thesis, University of Liège, Liège, Belgium.

Duchêne, L., Habraken, A.M., 2005. Analysis of the sensitivity of FEM predictions to numerical parameters in deep drawing simulations Eur. J. Mech. A/Solids 24, 614-629.

Duchêne, L., Habraken, A.M., Godinas, A., 2000. Validation of a FEM model coupled with texture applied to deep drawing process. In Proceedings of 3rd Int. Confi: ESAFORM'00, Stuttgart.

Duchêne, L., Godinas, A., Cescotto, S., Habraken, A.M., 2002. Texture evolution during deep-drawing processes. J. Mater. Process. Technol., 110-118.

Duchêne, L., Delannay, L., Habraken, A.M., 2004. Finite element prediction of the Swift effect based on Taylor-type polycrystal plasticity models. In: Stören, S. (Ed.), Proceedings of the 7th Esaform Conference on Material Forming, Trondheim, p. 171.

Duchêne, L., de Montleau, P., El Houdaigui, F., Bouvier S., Habraken A.M., 2005a. Analysis of texture evolution and hardening behavior during deep drawing with an improved mixed type FEM element. In: Smith, L.M., Pourboghrat, F., Yoon, J-W., Stoughton, T.B. (Eds.), Proceedings of Int. Conf. NUMISHEET 2005, Melville (New York), 1, pp. 409-414.

Duchêne, L., El Houdaigui, F., Habraken A.M., 2005b. Finite element simulations of the Swift effect. In: Bariani, P.F. (Ed.), Proceedings of the 8th Int. Conf. on Technology of Plasticity, Padova (Italy).

Habraken, A.M., Bourdouxhe, M., 1992. Coupled thermo-mechanical-metallurgical analysis during the cooling of steel pieces. Eur. J. Mech. A/Solids 11 (3), 381-402 
Habraken, A.M., Cescotto, S., 1990. An automatic remeshing technique for finite element simulation of forging processes. Int. J. Numer. Methods Engrg. 30, 1503-1525.

Habraken, A.M., Cescotto, S., 1998. Contact between deformable solids, the fully coupled approach. Math. Comput. Modell. 28 (4-8), 153169.

Habraken, A.M., Duchêne, L., 2004. Anisotropic elasto-plastic finite element analysis using a stress-strain interpolation method based on a polycrystalline model. Int. J. Plasticity 20 (8-9), 1525-1560.

Habraken, A.M., Charles, J.F., Wegria, J., Cescotto, S., 1998. Dynamic recrystallization during zinc rolling. Int. J. Form. Process. 1. Hill, R., 1948. A theory of the yielding and plastic flow of anisotropic metals. Proc. R. Soc. Lond. A 193, 281-297.

Hughes, T.J.R., 1983. Theoretical foundation for large-scale computations of nonlinear material behaviour. In: Nemat-Nasser, S., Asaro, R.J., Hegemier, G.A. (Eds.), Proceedings Workshop on Theor. Foundation for Large-Scale Computations of Nonlinear Material Behaviour. Martinus Nijhoff, Publisher, Dordrecht, pp. 29-63.

Jonas, J.J., Shrivastava, S., Toth, L.S., 1998. The inverse Swift effect: experiments and theory. Acta Mater. 46, 51-60.

Khan, A.S., Lopez-Pamies, O., Kazmi, R., 2006. Thermo-mechanical large deformation response and constitutive modeling of viscoelastic polymers over a wide range of strain rates and temperatures. Int. J. Plasticity 22 (4), 581-601.

Li, K.P., Cescotto, S., 1997. An 8-node brick element with mixed formulation for large deformation analyses. Comput. Methods Appl. Mech. Engrg. 141, 157-204

Miller, M.P., McDowell, D.L., 1996a. Modeling large strain multiaxial effects in fcc polycrystals. Int. J. Plasticity 12 (7), 875-902.

Miller, M.P., McDowell, D.L., 1996b. The effect of stress-state on the large strain inelastic deformation behavior of 304L stainless steel. ASME J. Engrg. Mater. Technol. 118, 28-36.

Mosbah, P., Shima, S., Habraken, A.M., Charlier, R., 1999. Numerical simulation of compacting process of a multi-steeped part with comparisons to experiments. J. Japan Soc. Powder Powder Metall. 46, 696-704.

Munhoven, S., Habraken, A.M., 1995. Application of an anisotropic yield locus based on texture to a deep drawing simulation. In: Shen, S.E., Dawson, P.R. (Eds.), Proceedings of the International Conference NUMIFORM 95 (Simulation of Materials Processing: Theory Methods, and Applications). Balkema, Rotterdam.

Nadai, A., 1950. Theory of Flow and Fracture. McGraw-Hill, New York, p. 349. Ponthot, J.P., 2002. Unified stress update algorithms for the numerical simulation of large deformation elasto-plastic and elasto-viscoplastic processes. Int. J. Plasticity 18, 91-126.

Poynting, J.H., 1909. Proc. R. Soc. Lond. A 82, 546.

Poynting, J.H., 1912. Proc. R. Soc. Lond. A 86, 534.

Qods, F., Toth, L.S., Van Houtte, P., 2005. Modelling of length changes and textures during free end torsion of cylindrical bars. Mater. Sci. Forum 495-497, 1609-1614.

Rohatgi, A., Jonas, J.J., Shrivastava, S., 1995. Effect of stress-relief annealing on the inverse Swift effect in steel and iron. Script. Metal. Mater. 32 (5), 737-741

Rose, W., Stüwe, H.P., 1968. Z. Metall. 59, 396.

Shrivastava, S.C., Jonas, J. J., Canova, G., 1982. Equivalent strain in large deformation torsion testing: theoretical and practical considerations. J. Mech. Phys. Solids 30 (1-2), 75-90.

Simo, J.C., Hughes, T.J.R., 1986. On the variational foundations of assumed strain methods. J. Appl. Mech., ASME 53, 51-54.

Swift, H.W., 1947. Length changes in metals under torsional overstrain. Engineering 163, 253-257.

Toth, L.S., Jonas, J. J., 1989. Analytic prediction of texture and length changes during free end torsion. Textures Microstruct. 10, $195-209$.

Toth, L.S., Gilormini, P., Jonas, J.J., 1988. Effect of rate sensitivity on the stability of torsion textures. Acta Metall. 36, $3077-3091$.

Toth, L.S., Jonas, J.J., Gilormini, P., Bacroix, B., 1990. Length changes during free end torsion: a rate sensitive analysis. Int. J. Plasticity 6, 83-108.

Toth, L.S., Szaszvari, P., Kovacs, I., Jonas, J.J., 1991. Shortening behaviour of drawn and twisted copper wires. Mater. Sci. Technol. 7, 458464. 
Toth, L.S., Jonas, J.J., Daniel, D., Bailey, J.A., 1992. Texture development and length changes in copper bars subjected to free end torsion. Textures Microstruct. 19, 245-262.

Toth, L.S., Qods, F., Fundenberger, J.-J., 2005. Modeling of axial strain in free end torsion of textured copper. Z. Metall. 96 (9), $1038-1043$.

Van Houtte, P., 1994. The 'MTM-FHM' software system, version 2, user's manual, KUL, Leuven.

Van Houtte, P., Li, S., Seefeldt, M., Delannay, L., 2005. Deformation texture prediction: from the Taylor model to the advanced Lamel model. Int. J. Plasticity 21 (3), 589-624.

Wang, J., Wagoner, R.H., 2004. A new hexahedral solid element for 3D FEM simulation of sheet metal forming. In: Ghosh, S., Castro, J.M., Lee, J.K. (Eds.), Proceedings of the 8th International Conference on Numerical Methods in Industrial Forming Processes, Columbus. AIP Conf. Proc. 712, 2181-2186.

Weber, L., Moser, B., Künzi, H.U., Mortensen, A., 2000. Swift and inverse Swift effect in alumina fiber reinforced aluminium wires. Acta Mater. 48, 2451-2459.

Wu, H.C., 2003. On finite plastic deformation of anisotropic metallic materials. Int. J. Plasticity 19, 91-119.

Wu, P.D., Neale, K.W., Van der Giessen, E., 1996. Simulation of the behaviour of fcc polycrystals during reversed torsion. Int. J. Plasticity $12(9), 1199-1219$.

Xiao, H., Bruhns, O.T., Meyers, A., 2001. Large strain responses of elastic-perfect plasticity and kinematic hardening plasticity with the logarithmic rate: Swift effect in torsion. Int. J. Plasticity 17, 211-235.

Zhu, Y.Y., Cescotto, S., 1994. Transient thermal and thermomechanical analysis by FEM. Comput. Struct. 53 (2), $275-304$.

Zhu, Y.Y., Cescotto, S., 1995. A fully coupled elasto-visco-plastic damage theory for anisotropic materials. Int. J. Solids Struct. 32 (11), $1607-1641$ 